\title{
Caracterización vibracional de piezocomposites metal-cerámica asimétricos
}

\author{
R. RUÍZ-BOULLOSA ${ }^{1}$, S. CORTÉS-FRAGOSO ${ }^{2}$, J. SANIGER-BLESA ${ }^{1}$, M.E. VILLAFUERTE-CASTREJÓN ${ }^{1}$, G. GONZÁLEZ², \\ P. OCHOA ${ }^{3}$, M. VILLEGAS ${ }^{3}$, Y J.F. FERNÁNDEZ ${ }^{3}$. \\ ${ }^{1}$ Centro de Instrumentos, Universidad Nacional Autónoma de México, Cd. Universitaria, México, D.F., 04510, México. \\ ${ }^{2}$ Instituto de Investigaciones en Materiales, Universidad Nacional Autónoma de México, Cd. Universitaria, México, D.F., 04510, México. \\ ${ }^{3}$ Departamento de Electrocerámica, Instituto de Cerámica y Vidrio, CSIC, 28500, Arganda del Rey, Madrid, España.
}

\begin{abstract}
Se han estudiado las características mecánico-vibracionales de piezocomposites metal-cerámica asimétricos. Kovar-PZT. Para esta caracterización se utilizó la técnica de vibrometría láser mediante la cual se obtienen perfiles de amplitud a diferentes frecuencias para el piezocomposite. Se muestran los modos diferenciados de vibración en el intervalo de $2 \mathrm{~Hz}$ a $20 \mathrm{kHz}$ y se discuten los resultados en la perspectiva de optimizar el diseño de los dispositivos.

Palabras clave: piezocomposite, vibración vibrometría láser.
\end{abstract}

\section{Vibrational characterictics of asymmetric metal-ceramic piezocomposites}

The mechanical and vibrational characteristics of asymmetric metal-ceramic piezocomposites, Kovar-PZT, have heen studied. For this characterization a laser vibrometry technique was used to obtain amplitude profiles of vibration at different frequencies for the piezocomposite. The vibration spectra showed modes in the range $2 \mathrm{~Hz}$ to $20 \mathrm{kHz}$. The results were discussed in the framework of design optimization.

Key words: piezocomposite, vibration spectra, laser vibrometry.

\section{INTRODUCCION}

El concepto de actuadores piezoeléctricos flexo-tensionales ha sido desarrollado recientemente (1-3) con el objeto de cubrir el vacío existente entre los actuadores bimorfos, capaces de producir grandes desplazamientos pero con limitaciones importantes en la generación de fuerza motriz, y el de los actuadores multicapa, capaces de generar fuerzas grandes y respuestas rápidas pero a costa de desplazamientos pequeños (4). Este nuevo concepto de actuadores encuentra por tanto aplicación cuando se requieren niveles intermedios de desplazamientos y de fuerzas motrices. El tipo de transductor estudiado en este trabajo, es un composite asimétrico metal-cerámica formado por un disco de PZT al que se fijó con resina epóxica en una de sus caras una cápsula metálica de kovar, conformada esta última de tal manera que queda una cavidad interna entre la cerámica y dicha cápsula $(3,5)$. De esta manera el movimiento radial de la cerámica piezoeléctrica genera un efecto flextensional en la cápsula metálica, originando un gran desplazamiento en la dirección perpendicular al disco cerámico. De esta manera resulta posible estudiar por separado las vibraciones de cada una de las caras. El estudio de la naturaleza de los diferentes modos vibracionales y sus implicaciones en el uso de estos piezocomposites como actuadores constituye el objetivo de este trabajo.

\section{METODOLOGIA EXPERIMENTAL}

\subsection{Fabricación del piezocomposite}

El dispositivo llamado címbal asimétrico consta de un disco piezoeléctrico de PZT y de una cápsula en forma de cono truncado sobre una de las caras del disco de PZT. El disco de PZT de $12.7 \mathrm{~mm}$ de diámetro y $1 \mathrm{~mm}$ de espesor, se obtuvo por reacción en estado sólido siguiendo el método cerámico (6) para el circonato titanato de plomo con la formulación equivalente a un PZT tipo PZT 5A (7). Los discos fueron tratados térmicamente a $400^{\circ} \mathrm{C}$ para disminuir esfuerzos internos, después se polarizaron, durante 15 minutos a $120^{\circ} \mathrm{C}$ dentro de un baño de silicona, con un campo eléctrico de $40 \mathrm{kV} / \mathrm{cm}$; a continuación se dejó enfriar el baño durante 30 minutos, hasta $80^{\circ} \mathrm{C}$, a partir de ahí se desconectó el campo eléctrico. Como material para las cápsulas se utilizó una lámina metálica de la aleación denominada kovar ( $54 \%$ Fe, $29 \%$ Ni, $17 \%$ co) de 250 $\mu \mathrm{m}$ de espesor. La forma final se obtuvo por troquelado aplicando una presión de $100 \mathrm{MPa}$, el diámetro de la cavidad es 8.7 mm y su espesor $270 \mathrm{~mm}$ (3). El disco cerámico de PZT y la cápsula se unieron a lo largo del perímetro con ayuda de una resina epoxíca. Las uniones se llevaron a cabo cuidadosamente evitando que se impregnara de manera accidental de la resina epoxíca. Para mejorar la unión, se aplicó una pequeña presión por medio de un pistón en las áreas de contacto. El espectro de impedancia del dispositivo se obtuvo con un analizador vectorial de impedancias HP4192A. 


\subsection{Sistema de medición}

Los dispositivos se recubrieron con un disco de papel retroreflectivo (22 $\mathrm{mg}$ de peso) que tiene la propiedad de reflejar la luz en la misma dirección de incidencia, tal como requiere el sistema de medición. Cada pastilla se sujetó por su borde con una gota pequeña de pegamento instantáneo a una cabeza esférica de alfiler de plástico, que a su vez se fijó al vástago de un posicionador óptico de dos dimensiones, posibilitándose así el desplazamiento del dispositivo a lo largo del plano XZ. De esta manera el dispositivo puede vibrar sin ninguna constricción, al estar suspendido únicamente de un punto.

Para la obtención de los modos de vibración se excitó la pastilla con la señal senoidal de un generador de funciones (HP 204) a diferentes frecuencias. El generador se conectó a su vez a un amplificador de audio (Yamaha AX380). Para proteger el amplificador se conectó una resistencia en paralelo con el dispositivo para disipar parte de la potencia generada. El voltaje promedio de excitación a la entrada de la pastilla fue de 20 Voltios rms.

El espectro de resonancia a frecuencias de audio, $20 \mathrm{~Hz}-20$ $\mathrm{kHz}$, se obtuvo midiendo en diversos puntos del disco la velocidad de vibración resultante al excitar a la pastilla con una señal eléctrica con un espectro de ruido blanco. Para medir la velocidad de vibración de las pastillas se utilizó un vibrómetro láser (B\&K 8323) que utiliza el efecto Doppler sobre la luz reflejada por una superficie vibrante, para obtener información de su velocidad de vibración. El haz de salida del instrumento se dirige a la superficie vibrante y la señal medida es la componente de velocidad del haz reflejado en la dirección de incidencia, por lo que se tiene que asegurar la perpendicularidad del haz con respecto a la superficie. La salida de voltaje del vibrómetro láser es proporcional a la velocidad de vibración. Este voltaje se conectó a un canal de un analizador digital de Fourier (B\&K 2034), mientras que en la otra entrada se conecta una derivación de la señal eléctrica de ruido blanco que, a través del amplificador de audio excita a la pastilla. El

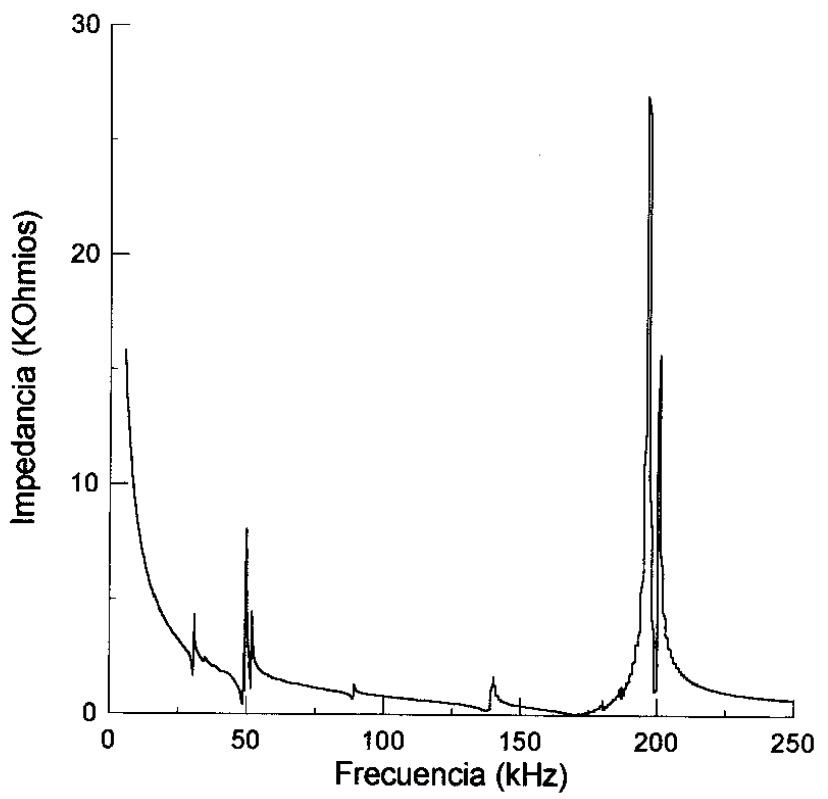

Figura 1. Espectro de resonancia mediante analizador vectorial de impedancias del cimbal asimétrico (tensión de oscilación de 1 voltio). analizador calcula la relación de los espectros de los dos canales para obtener el cociente entre velocidad y voltaje de excitación. Esta función está relacionada con la función de transferencia denominada admitancia mecánica (velocidad/fuerza) y presenta máximos en las resonancias mecánicas (la frecuencia de resonancia de velocidad se define como la frecuencia a la que la velocidad es un máximo).

En el caso de la obtención de las formas de vibración, el espectro de la señal vibratoria consta de una línea a la frecuencia de excitación. Se midió la amplitud del voltaje rms de esa línea, que es proporcional a la amplitud de la velocidad de vibración. Se obtuvieron los datos de la velocidad de vibración en una matriz de puntos sobre la superficie de la pastilla. Esta malla de puntos fue recorrida secuencialmente moviendo la pastilla, por pasos constantes en la dirección x y en la dirección z. De esta manera se obtuvo una matriz de datos de velocidad que corresponden punto a punto a la red o malla virtual de puntos sobre la pastilla. La gráfica en tres dimensiones de estos datos $\mathrm{v}(\mathrm{fn}, \mathrm{x}, \mathrm{z}$,$) para cada frecuencia de excitación fn nos$ da el módulo de la velocidad de vibración de la superficie a esa frecuencia. Al dividir la magnitud de la velocidad entre la frecuencia angular de excitación ( $2 p$ fn) se obtiene la magnitud (módulo) del desplazamiento (rms) de la superficie a esa frecuencia en determinado punto. Las gráficas se obtuvieron en Matlab haciendo una matriz para cada frecuencia de excitación y un pequeño programa para el despliegue de las mismas.

\section{RESULTADOS Y DISCUSION}

El espectro de impedancias de este dispositivo (figura 1) muestra un pico de resonancia, muy pequeño en $30,3 \mathrm{kHz}$ que corresponde al modo resonante del elemento metálico. Un sobretono de este modo de vibración aparece en $88,5 \mathrm{kHz}$. Los picos de resonancia en 48.5 y $138 \mathrm{kHz}$ son los correspondientes a los modos B02 y B04 de las resonancias de flexión, de acuerdo con los valores de las constantes de frecuencia, $2.46 \mathrm{y}$ 4.15 respectivamente (8). Finalmente aparece el pico más

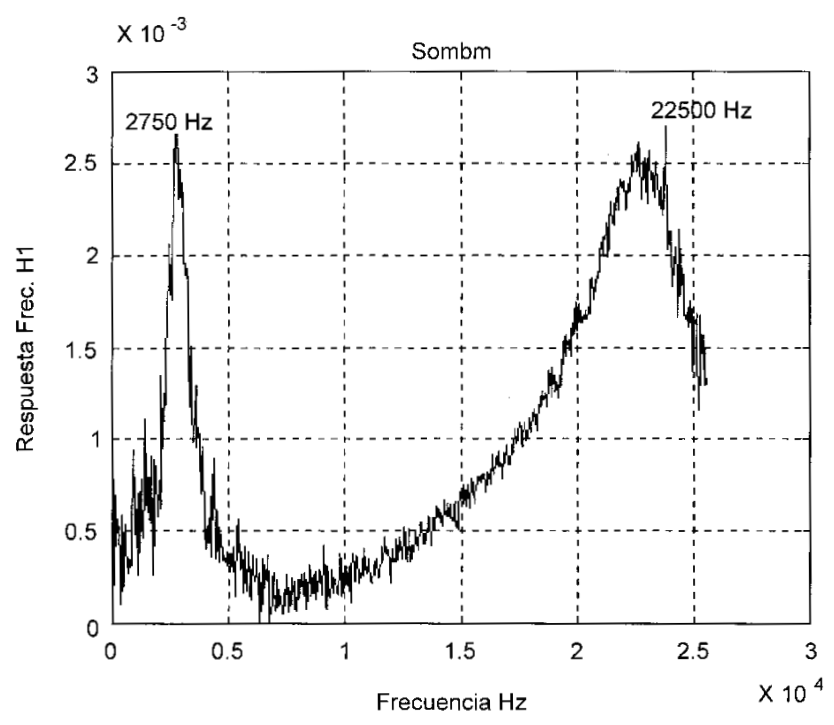

Figura 2. Espectro de resonancia mediante vibrometría láser del cimbal asimétrico (tensión de oscilación de 20 voltios). 
energético asociado al modo radial del PZT en 170.5 kHz. Para la frecuencia antiresonante del modo radial de vibración se observa un desdoblamiento que puede estar relacionado con la carga mecánica que supone la cápsula metálica, indicando la existencia de un gradiente de carga en la superficie del piezoeléctrico. Este desdoblamiento tiene su reflejo en el modo B02 de flexión. Por debajo de la frecuencia del elemento metálico no se espera la aparición de ningún pico de resonancia por lo que en consecuencia el piezocomposite debería seguir el campo aplicado y comportarse como un actuador. Sin embargo, al someter el dispositivo a frecuencias entre $2 \mathrm{~Hz}$ y $20 \mathrm{kHz}$ en el vibrómetro láser, se observó la emisión de señales audibles en el intervalo de $\sim 1 \mathrm{kHz}$ a $20 \mathrm{kHz}$ y en particular en dos zonas de frecuencia el sonido emitido aumentaba en intensidad (sensado auditivamente) lo que parecía indicar la existencia de resonancias de menor amplitud en esa zona espectral. Se procedió pues a hacer las mediciones mencionadas anteriormente para investigar si efectivamente había frecuencias en las que la amplitud de las vibraciónes fueran mayores.

En la figura 2 se muestra el espectro de vibración (velocidad amplitud de voltaje de excitación), del címbal asimétrico hasta $25 \mathrm{kHz}$. Se observa que en este intervalo la vibración del sistema está definida por dos picos de resonancia, el primero a $2.75 \mathrm{kHz}$ con una anchura aproximada de $0.1 \mathrm{kHz}$ y el segundo centrado en $22.5 \mathrm{kHz}$ con una anchura estimada de $5 \mathrm{kHz}$ (entre frecuencias donde la amplitud de la velocidad es igual a la mitad de la amplitud máxima). Dada la fuerte diferencia en la forma de los picos (semianchura) es de esperar que la forma de vibración del dispositivo esté marcada mayoritariamente por el pico de $22.5 \mathrm{kHz}$ y que el predominio del primero de ellos ocurra exclusivamente a frecuencias muy próximas a los $2.75 \mathrm{kHz}$. La explicación de porqué aparece este modo no es definitiva. Debido a la configuración experimental del dispositivo de medida, el primer modo de vibración podría estar relacionado con la fijación del dispositivo en la punta de un alfiler. De esta forma el dispositivo vibraría como una especie de cantilever anclado. Pero podría ser también que a esta frecuencia el metal y la pastilla se flexionen efectivamente con un modo de mariposa con una linea nodal. La reducción en frecuencia del modo de vibración correspondiente a la cápsula metálica podría estar relacionada con la carga de la película retrorefrectiva. La adhesión de un disco de $3 \mathrm{~mm}$ de diámetro y $28 \mathrm{mg}$ de masa en la parte superior del cono truncado de la cápsula metálica provoca una reducción del modo de vibración en 8 kHz en un címbal asimétrico (8).

Las figuras 3 y 4 muestran, con fines de comparación las formas de vibración del dispositivo por cada una de sus caras a diferentes frecuencias. Las amplitudes de vibración a 2.7, 8.0, 11.8 y $20 \mathrm{kHz}$ por la cara de la cápsula metálica de Kovar se presentan en la figura 3, con valores de amplitudes de vibración que van de 100 a $300 \mathrm{~nm}$ de manera creciente según su proximidad al máximo de resonancia en $22.5 \mathrm{kHz}$. En la imagen de la figura 3a se presenta el modo de vibración que pudiera denominarse de doble ala o de mariposa, con mayor amplitud en los bordes de la pastilla que en el centro. Para frecuencias superiores se observa un incremento de la amplitud junto con un centrado del desplazamiento en el dispositivo, lo que le confiere una forma de sombrero. Si se interpretan estas formas de vibración con base en el espectro de la figura 2, puede presuponerse una correlación del modo de vibración de doble ala, a $2.7 \mathrm{kHz}$, con el pico de resonancia de esa misma frecuencia. A mayores frecuencias el modo correspondería fundamentalmente a la influencia del modo de mayor semianchura centrado en $22.5 \mathrm{kHz}$.

En la figura 4 se presentan las amplitudes y formas de vibración del címbal asimétrico por el lado de la cerámica PZT. Nuevamente, el modo a $2.7 \mathrm{kHz}$ es del tipo doble ala lo que denota la influencia del primer pico de resonancia. Sin embargo, en las frecuencias siguientes de 8.0 y $11.8 \mathrm{kHz}$, se observa una doble tendencia. Por una parte, de manera similar a lo que se vio en la cara del kovar, va apareciendo un máximo relativo de amplitud en el centro de la pastilla, mientras que por el otro lado se conservan los valores máximos de amplitud en los bordes de la pastilla. A lo anterior se añade la formación de una zona vibracional de forma circular que separa las zonas vibrantes del borde y centro de la pastilla, de menor amplitud,

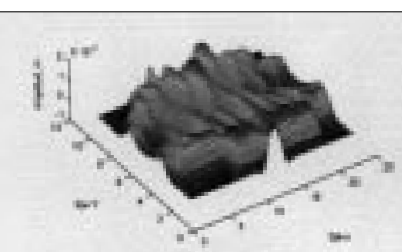

a)

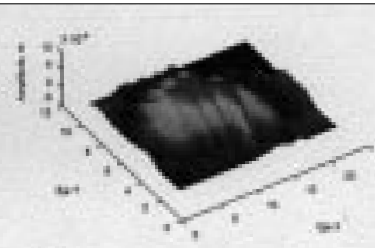

b)

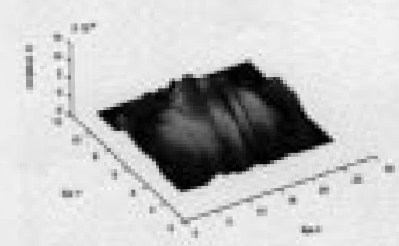

c)

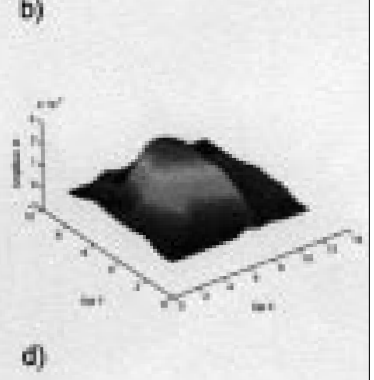

Figura 3. Modos de vibración del cimbal asimétrico por la cara del kovar, (a) $2.7 \mathrm{kHz}$, (b) $8.0 \mathrm{kHz}$, (c) $11.8 \mathrm{kHz}$ y (d) $20 \mathrm{kHz}$.

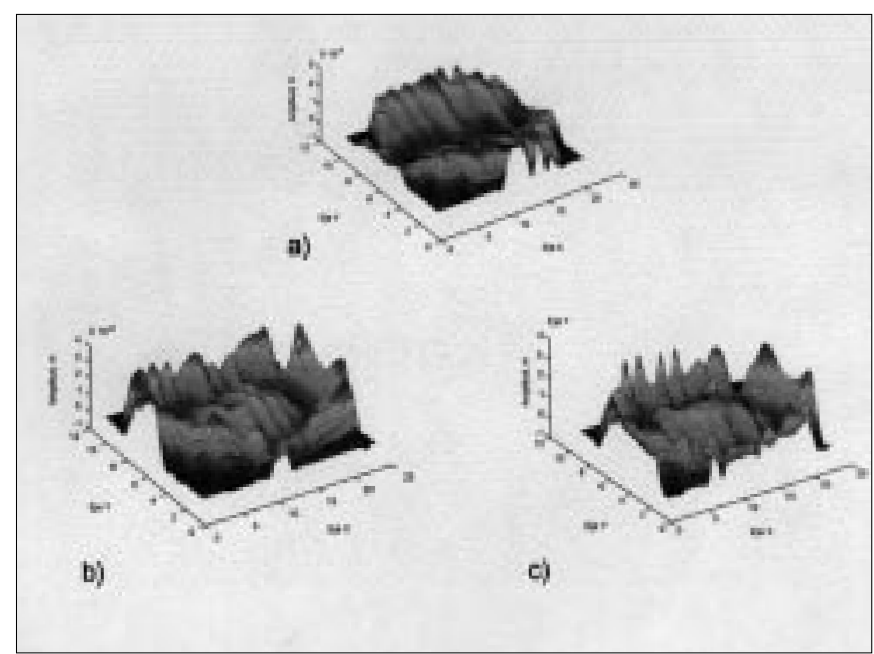

Figura 4. Modos de vibración del cimbal asimétrico por la cara del PZT, (a) $2.7 \mathrm{kHz}$, (b) $8.0 \mathrm{khz}$ y (c) $11.8 \mathrm{kHz}$. 
probablemente producida por la rigidez de la cápsula en esta zona y la resina epóxica. La forma y dimensión de esta zona de poca amplitud sugiere una sociación con la estructura de kovar en la cara opuesta. La forma de la estructura metálica y su rigidez mecánica anclan esta zona en la cara opuesta .

\section{CONCLUSIONES}

La técnica de vibrometría láser se manifiesta como una herramienta útil para estudiar los modos vibracionales de los dispositivos PZT-kovar en el intervalo de frecuencias audibles.

Se pueden distinguir dos modos de vibración en el intervalo espectral estudiado. La primera denominada de doble ala o de mariposa, está ligada al pico de resonancia de menor frecuencia, $2.75 \mathrm{kHz}$, en el espectro de resonancia de la velocidad y esta asociado probablemente a una vibración del dispositivo en modo cantilever. El segundo modo, que produce amplitudes de vibración crecientes desde el borde hasta el centro de la pastilla, se manifiesta en un intervalo más amplio de frecuencias en consonancia con la semianchura de la resonancia a la que se asocia. Este modo está asociado a la vibración de la cápsula metálica y ocurre a $22.5 \mathrm{kHz}$ en el espectro de velocidad.

Finalmente, el estudio de los modos vibracionales por el lado de la cerámica revela la influencia de esta estructura sobre el dispositivo. La rigidez mecánica de la cápsula metálica se transmite a través del espesor de la pastilla cerámica, produciendo una zona de relativa poca amplitud en forma de anillo en la cerámica.

\section{AGRADECIMIENTOS}

Los autores agradecen el apoyo financiero para la realización de esta investigación a la Universidad Nacional Autónoma de México, Consejo Nacional de Ciencia y Tecnología de México, Comisión Internacional de Ciencia y Tecnología de España y Programa para la Cooperación y el Desarrollo a través de los proyectos PAPIIT-IN106998, CONACYT-27624U, CICYT MAT97-694-C02-01 y CYTED Red VIII.F.

\section{BIBLIOGRAFÍA}

1. R.E. Newnham, Q.C. Xu, S. Yoshikawa. "Transformed stress direction-acoustic transducer". U.S. Patent No. 4999819 (12 March 1992).

2. J.F. Fernández, A. Dogan, Q.M. Zhang, J.F. Tressler, R.E. Newnham. “Hollow piezoelectric composites". Sensors and Actuators A 51,183-92 (1996).

3. J.F. Fernández, A. Dogan, J.T. Fielding, K. Uchino, R.E. Newnham. “Tailoring the performance of ceramic-metl piezocomposite actuators, "cymbals""." Sensors and Actuators A 65,228-37 (1998).

4. K. Uchino, Piezoelectric/electrostirictive actuators. Morikita Publishers Tokyo 1996.

5. Q.C. Xu, A. Dogan, J. Tressler, S. Yoshikawa, R.E. Newnham, “Ceramic Metalcomposite actuators". Ferroelectrics, 160 (1994) 337-46.

6. L. Celi, A.C. Caballero, M. Villegas, P. Durán, C. Moure, J.F. Fernández. "Efecto de las características de los polvos cerámicos sobre la densificación y propiedades de materiales cerámicos tipo PZT". Bol. Soc. Esp. Ceram. Vidrio. 38 [5] 493-497 (1999)

7. Morgan Matroc S.A. Catalogo de productos. Bedford, OH, USA.

8. S. Ueda, Y. Tomikawa. Ultrasonic Motors, Theory and Applications. Oxford Science Publications, Clarendon Press, Oxford 1993.

9. P. Leidinger. Tunability of Cymbals as piezo-metal transducers. Diplomarbeit Thesis. Instituto de Cerámica y Vidrio 1996. 